ethical review and potential participants' recruitment is underway.

Conclusions of 132 quality indicators for ACP/GCD, 9 are feasible, valid, usable and acceptable for monitoring performance in the rollout of ACP/GCD. This set of indicators shows promise for describing and evaluating ACP/GCD uptake throughout a complex, multi-sector healthcare system.

\section{OP83 THE CHALLENGE OF FINDING THE 'RIGHT' OUTCOME TO MEASURE THE EFFECTS OF ACP - PART I}

1J Rietjens*, ${ }^{2} \mathrm{R}$ Sudore, ${ }^{1} \mathrm{~A}$ van der Heide, ${ }^{3} \mathrm{D}$ Heyland, ${ }^{1} \mathrm{I}$ Korfage. ${ }^{1}$ Erasmus University Medical Centre, Rotterdam, Netherlands; ${ }^{2}$ University of California, San Fransisco, USA; ${ }^{3}$ Queen's University, Kingston, Canada

10.1136/spcare-2019-ACPICONGRESSABS.83

Background Advance care planning (ACP) is increasingly implemented in oncology and beyond, but a definition of ACP and recommendations concerning its use are lacking. This hinders the development of ACP programmes and the evaluation of ACP's effectiveness.

Methods We used a formal Delphi consensus process to help develop a definition of ACP and provide recommendations for its application in healthcare, policy and research.

Results Of the 109 experts (82 from Europe, 16 from North America, and 11 from Australia) who rated the ACP definitions and its 41 recommendations, agreement for each definition or recommendation was between $68-100 \%$. ACP was defined as the ability to enable individuals to define goals and preferences for future medical treatment and care, to discuss these goals and preferences with family and health-care providers, and to record and review these preferences if appropriate. Recommendations included the adaptation of ACP based on the readiness of the individual; targeting ACP content as the individual's health condition worsens; and, using trained non-physician facilitators to support the ACP process. A list of outcome measures is also presented to enable the pooling and comparison of results of ACP studies.

Conclusion This large international Delphi panel was able to come to a consensus on an ACP definition and recommendations. This represents an important first step in providing clarity with a view to further policy and research in this field. We believe that our recommendations can provide guidance for clinical practice, ACP policy, and research.

\section{OP84 THE CHALLENGE OF FINDING THE 'RIGHT' OUTCOME TO MEASURE THE EFFECTS OF ACP - PART II}

${ }^{1} \mathrm{R}$ Sudore, ${ }^{2} \mathrm{D}$ Heyland, ${ }^{3} \mathrm{H}$ Lum, ${ }^{4} \mathrm{~J}$ Rietjens, ${ }^{4} \mathrm{~K}$ Korfage ${ }^{*},{ }^{5} \mathrm{M}$ Howard, ${ }^{5} \mathrm{~J}$ You. ${ }^{1}$ University of California, San Fransisco, USA; ${ }^{2}$ Queen's University, Kingston, Canada; ${ }^{3}$ Denver, Colorado, USA; ${ }^{4}$ Erasmus University Medical Center, Rotterdam, Netherlands; ${ }^{5}$ McMaster University, Hamilton, Ontario, Canada

\subsection{6/spcare-2019-ACPICONGRESSABS.84}

Background Standardized outcomes that define successful advance care planning (ACP) are lacking. The objective of this study was to create an Organizing Framework of ACP outcome constructs and rate the importance of these outcomes.
Methods This study convened a Delphi panel consisting of 52 multidisciplinary, international ACP experts including clinicians, researchers, and policy leaders from four countries. We conducted literature reviews and solicited attendee input from five international ACP conferences to identify initial ACP outcome constructs. In five Delphi rounds, we asked panelists to rate patient-centered outcomes on a seven-point "not-at-all" to "extremely important" scale. We calculated means and analyzed panelists' input to finalize an Organizing Framework and outcome rankings.

Results Organizing Framework outcome domains included process (e.g., attitudes), actions (e.g., discussions), quality of care (e.g., satisfaction), and health care (e.g., utilization). The top five outcomes included 1) care consistent with goals, mean 6.71 ( \pm SD 0.04); 2) surrogate designation, 6.55 (0.45); $3)$ surrogate documentation, $6.50(0.11)$; 4) discussions with surrogates, 6.40 (0.19); and 5) documents and recorded wishes are accessible when needed 6.27 (0.11). Advance directive documentation was ranked 10th, 6.01 (0.21). Panelists raised caution about whether "care consistent with goals" can be reliably measured.

Conclusion A large, multidisciplinary Delphi panel developed an Organizing Framework and rated the importance of ACP outcome constructs. Top rated outcomes should be used to evaluate the success of ACP initiatives. More research is needed to create reliable and valid measurement tools for the highest rated outcomes, particularly "care consistent with goals".

\section{OP85 THE CHALLENGE OF FINDING THE 'RIGHT' OUTCOME TO MEASURE THE EFFECTS OF ACP: PART III}

S Hickman* , K Unroe, A Torke. Indiana University, Indianapolis, USA

\subsection{6/spcare-2019-ACPICONGRESSABS.85}

Background A key outcome of successful advance care planning is consistency between patient goals of care and the care provided. However, assessing consistency is methodologically challenging due to conceptual and logistical barriers as well as practice limitations.

Methods The team reviewed the literature and identified key methodological barriers to measuring care consistency with patient preferences and goals of care. Strategies to overcome these barriers are proposed.

Results Key methodological barriers include: 1) the specificity and relevance of documentation about preferences and goals of care; 2) the availability of documentation about preferences and goals of care; 3 ) the stability of documented preferences and goals of care; 4) the timing of treatments provided and identifying decisions not to treat ("nonevents"); and 5) calculating rates of consistency. Strategies to address these methodological barriers include using tools to document specific preferences, incorporation into electronic health records, period reassessment of preferences, prospective data collection, and using percent agreement to report consistency.

Discussion There are clear challenges to assessing care consistency with preferences and goals of care. The ability to assess and report on this important outcome of successful advance care planning requires focused efforts to improve practice. 